(C) 2008 The Japan Society of Applied Physics

\title{
On-Wafer Characterization of Thermomechanical Properties of Isotropic Thin Films Deposited on Anisotropic Substrates
}

\author{
Liang-Chieh WU and Yuan-Fang CHOU* \\ Department of Mechanical Engineering, National Taiwan University, Taipei, Taiwan 10617, Republic of China
}

(Received December 29, 2007; accepted March 21, 2008; published online July 11, 2008)

An on-wafer characterization technique has been developed to determine the thermomechanical properties of a thin film. The thin film is deposited on an anisotropic substrate with in-plane principal directions of elasticity. The mismatch of thermomechanical properties between the substrate and the thin film causes the bilayer structure to deflect. Measuring this deflection and fitting it with a surface equation gives curvatures of the bilayer structure. The relationships between curvatures and thermomechanical properties are derived from equilibrium conditions. The results of a sensitivity analysis suggest the adoption the Poisson's ratio of bulk material such that the Young's modulus and the coefficient of thermal expansion (CTE) of the thin film can be found from fitted principal curvatures. An aluminum thin film deposited on an ST-cut quartz substrate at high temperature is employed for verification. The Young's modulus and CTE of the aluminum film are found to be 81.25 GPa and $25.52 \mathrm{ppm} /{ }^{\circ} \mathrm{C}$, respectively. Data analysis shows that the characterization of Young's modulus and CTE is much more sensitive to principal curvatures than the Poisson's ratio. [DOI: 10.1143/JJAP.47.5623]

KEYWORDS: thermomechanical properties, thin film, substrate, anisotropic, curvature

\section{Introduction}

Thin films are often adopted as materials for microstructures in rapidly growing microelectromechanical system (MEMS) devices. The thermomechanical properties of thin films are indispensable in designing and manipulating microstructures. Due to the thicknesses of thin films generally being in the nanometer range, their thermomechanical properties might be different from those of bulk materials. Therefore, the bulk material properties might not be applicable anymore and developing a reliable characterization method is indispensable.

To characterize the mechanical properties of thin films, different testing methods were developed by many researchers. In 1988, Johansson et al. first determined Young's modulus from the bending test of a silicon microcantilever beam. ${ }^{1)}$ This test is based on the linear bending theory in which the measurements of the applied force and beam deflection are required. Using an atomic force microscope, Sundararajan et al. conducted the same bending test on a nanoscale clamped-clamped beam in 2002. ${ }^{2)}$ They determined Young's moduli of silicon and silicon dioxide. Allen et al. developed the bulging test of a polyimide membrane in 1987.3) The bulging deflection under uniform pressure is measured to determine Young's modulus and residual stress. Compared with indirect methods, such as beam bending and bulging test, direct tensile testing on a micromachined polysilicon specimen was conducted by Sharpe et al. in 1997. ${ }^{4}$ In addition to measuring the applied load, they measured strain by laser-based interferometry to evaluate Young's modulus and fracture strength. Using a nanoindenter, Son et al. carried out bending tests of aluminum and gold microcantilever beams in 2003. ${ }^{5)}$ Young's moduli and yield strengths of beam materials were estimated and their dependence on the grain size was studied. In 1979, Petersen and Guarnieri designed a frequency sensing technique for electrostatically vibrating microcantilever beams. ${ }^{6}$ ) Based on the known mass densities and beam geometries, they extracted Young's moduli of various

*E-mail address: yfchou@ntu.edu.tw insulating thin films from the measured resonant frequencies. Other than the mechanical properties, the thermomechanical properties of thin films could also be determined by taking into account the thermal mismatch between the thin film and the substrate. In 1980, Retajczyk and Sinha employed the relationships between thermal stresses and temperature variations to evaluate elastic biaxial moduli and coefficients of thermal expansion (CTE) of $\mathrm{BN}$ and $\mathrm{B}_{2} \mathrm{~N}$ thin films deposited on silicon and fused quartz substrates. ${ }^{7)} \mathrm{By}$ combing the experimental and finite element analysis results, Zhao et al. improved this method to determine the Poisson's ratio of silicon dioxide films on silicon and GaAs substrates in 1999. ${ }^{8)}$ In 2000, Zhao et al. deposited the same thin films on two substrates, one isotropic and the other anisotropic, to simultaneously evaluate the Young's modulus, Poisson's ratio and CTE of the thin films. ${ }^{9}$ )

In this study, a novel on-wafer technique for the characterization of thermomechanical properties of thin films is developed. The deflection of the film-substrate-formed bilayer structure is the only data required. Based on equilibrium conditions, a mathematical model describing relationships between curvatures and thermomechanical properties is established. An aluminum thin film deposited on an ST-cut quartz substrate is employed for verification. Measuring the deflection of the bilayer structure and fitting it with a surface equation yield the induced curvatures from which the Young's modulus and CTE of the aluminum thin film are evaluated.

\section{Mathematical Model}

A film-substrate bilayer structure is formed by depositing a thin film of thickness $t_{\mathrm{f}}$ onto a substrate of thickness $t_{\mathrm{s}}$, as shown in Fig. 1. As the deposition temperature is higher than room temperature by $\Delta T$, the mismatch in CTE between the film and the substrate causes the bilayer structure to bend. Moreover, for an anisotropic substrate, twisting other than bending may also be generated. The lower the symmetry of the substrate, the more complex deformation is resulted. Therefore, a general expression for substrates of low symmetry should be derived such that most information can be explored. 


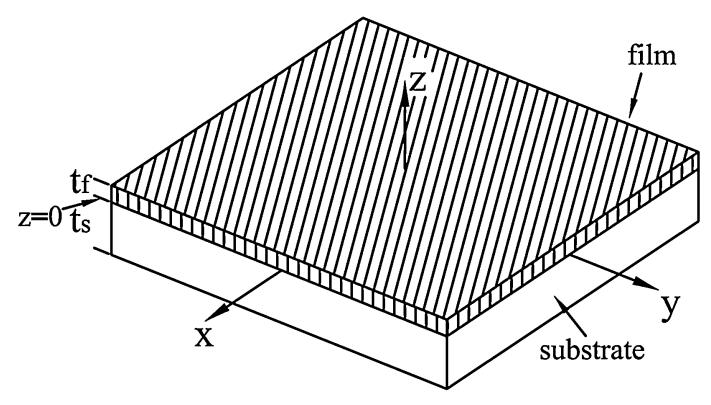

Fig. 1. Schematic diagram of film-substrate structure.

The in-plane strains $\varepsilon_{x}, \varepsilon_{y}$, and $\varepsilon_{x y}$ in the bilayer structure can be treated as a combination of two parts. The first part consists of the midplane strains $\varepsilon_{\mathrm{m} x}, \varepsilon_{\mathrm{m} y}$, and $\varepsilon_{\mathrm{m} x y}$, whereas strains due to the midplane curvatures $\kappa_{x}$ and $\kappa_{y}$ and the midplane twist $\kappa_{x y}$ compose the second part. The in-plane strains therefore can be expressed as

$$
\begin{aligned}
\varepsilon_{x} & =\varepsilon_{\mathrm{m} x}-\left(z-z_{\mathrm{m}}\right) \kappa_{x}, \\
\varepsilon_{y} & =\varepsilon_{\mathrm{m} y}-\left(z-z_{\mathrm{m}}\right) \kappa_{y}, \\
\varepsilon_{x y} & =\varepsilon_{\mathrm{m} x y}-\left(z-z_{\mathrm{m}}\right) \kappa_{x y},
\end{aligned}
$$

where $z_{\mathrm{m}}$ is the midplane location and $z$ is the distance measured from the interface. For a thin plate, it is reasonable to neglect the out-of-plane stresses; therefore, the normal stresses $\sigma_{x}$ and $\sigma_{y}$ and the shear stress $\tau_{x y}$ compose a state of plane stress at any distance $z$. Consequently, Hooke's law becomes

$$
\begin{gathered}
\varepsilon_{x}=s_{11} \sigma_{x}+s_{12} \sigma_{y}+s_{16} \tau_{x y}+\alpha_{x} \Delta T, \\
\varepsilon_{y}=s_{12} \sigma_{x}+s_{22} \sigma_{y}+s_{26} \tau_{x y}+\alpha_{y} \Delta T, \\
2 \varepsilon_{x y}=s_{16} \sigma_{x}+s_{26} \sigma_{y}+s_{66} \tau_{x y}+2 \alpha_{x y} \Delta T,
\end{gathered}
$$

where $s_{11}, s_{22}, s_{12}, s_{16}, s_{26}$, and $s_{66}$ are the matrix notations of elastic compliances $s_{i j k l}$. For an isotropic thin film, the stresses in the film can be obtained from eqs. (2)-(4) as

$$
\begin{aligned}
\sigma_{x}^{\mathrm{f}} & =B_{\mathrm{f}}\left[\left(\varepsilon_{x}-\alpha_{\mathrm{f}} \Delta T\right)+v_{\mathrm{f}}\left(\varepsilon_{y}-\alpha_{\mathrm{f}} \Delta T\right)\right], \\
\sigma_{y}^{\mathrm{f}} & =B_{\mathrm{f}}\left[\left(\varepsilon_{y}-\alpha_{\mathrm{f}} \Delta T\right)+v_{\mathrm{f}}\left(\varepsilon_{x}-\alpha_{\mathrm{f}} \Delta T\right)\right], \\
\tau_{x y}^{\mathrm{f}} & =2 G_{\mathrm{f}} \varepsilon_{x y},
\end{aligned}
$$

with $B_{\mathrm{f}}=E_{\mathrm{f}} /\left(1-v_{\mathrm{f}}^{2}\right)$, where the superscript $\mathrm{f}$ denotes the film, $E_{\mathrm{f}}, G_{\mathrm{f}}, v_{\mathrm{f}}$, and $\alpha_{\mathrm{f}}$ refer to the Young's modulus, shear modulus, Poisson's ratio, and CTE of the film. The stresses in the substrate obtained from eqs. (2)-(4) are

$$
\begin{aligned}
\sigma_{x}^{\mathrm{s}}= & B_{11}\left(\varepsilon_{x}-\alpha_{x}^{\mathrm{s}} \Delta T\right)+B_{12}\left(\varepsilon_{y}-\alpha_{y}^{\mathrm{s}} \Delta T\right) \\
& +2 B_{16}\left(\varepsilon_{x y}-\alpha_{x y}^{\mathrm{s}} \Delta T\right), \\
\sigma_{y}^{\mathrm{s}}= & B_{12}\left(\varepsilon_{x}-\alpha_{x}^{\mathrm{s}} \Delta T\right)+B_{22}\left(\varepsilon_{y}-\alpha_{y}^{\mathrm{s}} \Delta T\right) \\
& +2 B_{26}\left(\varepsilon_{x y}-\alpha_{x y}^{\mathrm{s}} \Delta T\right), \\
\tau_{x y}^{\mathrm{s}}= & B_{16}\left(\varepsilon_{x}-\alpha_{x}^{\mathrm{s}} \Delta T\right)+B_{26}\left(\varepsilon_{y}-\alpha_{y}^{\mathrm{s}} \Delta T\right) \\
& +2 B_{66}\left(\varepsilon_{x y}-\alpha_{x y}^{\mathrm{s}} \Delta T\right),
\end{aligned}
$$

where the material constants $B_{p q}, p, q=1,2, \ldots, 6$, are defined as

$$
\begin{aligned}
& B_{11}=1 / \Delta\left(s_{22} s_{66}-s_{26}^{2}\right), \\
& B_{22}=1 / \Delta\left(s_{11} s_{66}-s_{16}^{2}\right), \\
& B_{66}=1 / \Delta\left(s_{11} s_{22}-s_{12}^{2}\right),
\end{aligned}
$$

$$
\begin{aligned}
& B_{12}=1 / \Delta\left(s_{16} s_{26}-s_{12} s_{66}\right), \\
& B_{16}=1 / \Delta\left(s_{12} s_{26}-s_{22} s_{16}\right), \\
& B_{26}=1 / \Delta\left(s_{12} s_{16}-s_{11} s_{26}\right),
\end{aligned}
$$

with

$$
\Delta=\left|\begin{array}{lll}
s_{11} & s_{12} & s_{16} \\
s_{12} & s_{22} & s_{26} \\
s_{16} & s_{26} & s_{66}
\end{array}\right|,
$$

and the superscript $\mathrm{s}$ denotes the substrate, and $\alpha_{x}^{\mathrm{s}}, \alpha_{y}^{\mathrm{s}}$, and $\alpha_{x y}^{\mathrm{s}}$ are the in-plane CTEs of the substrate.

In the absence of external force and moment, the bilayer structure has to satisfy the force equilibrium as

$$
\begin{aligned}
& \int_{-t_{\mathrm{s}}}^{0} \sigma_{x}^{\mathrm{s}} d z+\int_{0}^{t_{\mathrm{f}}} \sigma_{x}^{\mathrm{f}} d z=0 \\
& \int_{-t_{\mathrm{s}}}^{0} \sigma_{y}^{\mathrm{s}} d z+\int_{0}^{t_{\mathrm{f}}} \sigma_{y}^{\mathrm{f}} d z=0 \\
& \int_{-t_{\mathrm{s}}}^{0} \tau_{x y}^{\mathrm{s}} d z+\int_{0}^{t_{\mathrm{f}}} \tau_{x y}^{\mathrm{f}} d z=0
\end{aligned}
$$

and the moment equilibrium as

$$
\begin{gathered}
\int_{-t_{\mathrm{s}}}^{0} \sigma_{x}^{\mathrm{s}}\left(z-z_{\mathrm{m}}\right) d z+\int_{0}^{t_{\mathrm{f}}} \sigma_{x}^{\mathrm{f}}\left(z-z_{\mathrm{m}}\right) d z=0, \\
\int_{-t_{\mathrm{s}}}^{0} \sigma_{y}^{\mathrm{s}}\left(z-z_{\mathrm{m}}\right) d z+\int_{0}^{t_{\mathrm{f}}} \sigma_{y}^{\mathrm{f}}\left(z-z_{\mathrm{m}}\right) d z=0, \\
\int_{-t_{\mathrm{s}}}^{0} \tau_{x y}^{\mathrm{s}}\left(z-z_{\mathrm{m}}\right) d z+\int_{0}^{t_{\mathrm{f}}} \tau_{x y}^{\mathrm{f}}\left(z-z_{\mathrm{m}}\right) d z=0 .
\end{gathered}
$$

Substituting eq. (1) and eqs. (5)-(10) into eqs. (12)-(14), the force equilibrium can be separated into two parts. Firstly, the resultant forces induced by the curvatures and twist are zero, that is,

$$
\begin{gathered}
\left(B_{11} \kappa_{x}+B_{12} \kappa_{y}+2 B_{16} \kappa_{x y}\right)\left(\frac{t_{\mathrm{s}}^{2}}{2}+z_{\mathrm{m}} t_{\mathrm{s}}\right) \\
-B_{\mathrm{f}}\left(\kappa_{x}+v_{\mathrm{f}} \kappa_{y}\right)\left(\frac{t_{\mathrm{f}}^{2}}{2}-z_{\mathrm{m}} t_{\mathrm{f}}\right)=0, \\
\left(B_{12} \kappa_{x}+B_{22} \kappa_{y}+2 B_{26} \kappa_{x y}\right)\left(\frac{t_{\mathrm{s}}^{2}}{2}+z_{\mathrm{m}} t_{\mathrm{s}}\right) \\
-B_{\mathrm{f}}\left(\kappa_{y}+v_{\mathrm{f}} \kappa_{x}\right)\left(\frac{t_{\mathrm{f}}^{2}}{2}-z_{\mathrm{m}} t_{\mathrm{f}}\right)=0, \\
\left(B_{16} \kappa_{x}+B_{26} \kappa_{y}+2 B_{66} \kappa_{x y}\right)\left(\frac{t_{\mathrm{s}}^{2}}{2}+z_{\mathrm{m}} t_{\mathrm{s}}\right) \\
-2 G_{\mathrm{f}} \kappa_{x y}\left(\frac{t_{\mathrm{f}}^{2}}{2}-z_{\mathrm{m}} t_{\mathrm{f}}\right)=0 .
\end{gathered}
$$

Secondly, the resultant forces caused by the midplane strains are also zero, which results in

$$
\begin{aligned}
& {\left[B_{11}\left(\varepsilon_{\mathrm{m} x}-\alpha_{x}^{\mathrm{s}} \Delta T\right)+B_{12}\left(\varepsilon_{\mathrm{m} y}-\alpha_{y}^{\mathrm{s}} \Delta T\right)\right.} \\
& \left.\quad+2 B_{16}\left(\varepsilon_{\mathrm{m} x y}-\alpha_{x y}^{\mathrm{s}} \Delta T\right)\right] t_{\mathrm{s}} \\
& \quad+B_{\mathrm{f}}\left[\left(\varepsilon_{\mathrm{m} x}-\alpha_{\mathrm{f}} \Delta T\right)+v_{\mathrm{f}}\left(\varepsilon_{\mathrm{m} y}-\alpha_{\mathrm{f}} \Delta T\right)\right] t_{\mathrm{f}}=0 \\
& {\left[B_{12}\left(\varepsilon_{\mathrm{m} x}-\alpha_{x}^{\mathrm{s}} \Delta T\right)+B_{22}\left(\varepsilon_{\mathrm{m} y}-\alpha_{y}^{\mathrm{s}} \Delta T\right)\right.} \\
& \left.\quad+2 B_{26}\left(\varepsilon_{\mathrm{m} x y}-\alpha_{x y}^{\mathrm{s}} \Delta T\right)\right] t_{\mathrm{s}} \\
& \quad+B_{\mathrm{f}}\left[\left(\varepsilon_{\mathrm{m} y}-\alpha_{\mathrm{f}} \Delta T\right)+v_{\mathrm{f}}\left(\varepsilon_{\mathrm{m} x}-\alpha_{\mathrm{f}} \Delta T\right)\right] t_{\mathrm{f}}=0 \\
& \quad\left[B_{16}\left(\varepsilon_{\mathrm{m} x}-\alpha_{x}^{\mathrm{s}} \Delta T\right)+B_{26}\left(\varepsilon_{\mathrm{m} y}-\alpha_{y}^{\mathrm{s}} \Delta T\right)\right. \\
& \left.\quad+2 B_{66}\left(\varepsilon_{\mathrm{m} x y}-\alpha_{x y}^{\mathrm{s}} \Delta T\right)\right] t_{\mathrm{s}}+2 G_{\mathrm{f}} \varepsilon_{\mathrm{m} x y} t_{\mathrm{f}}=0 .
\end{aligned}
$$


Providing that the thin film is much thinner than the substrate, i.e., $t_{\mathrm{f}} \ll t_{\mathrm{s}}$, the midplane location can be approximated as $z_{\mathrm{m}}=-t_{\mathrm{s}} / 2$ from eqs. (18)-(20). Employing this $z_{\mathrm{m}}$ and substituting eq. (1) and eqs. (5)-(10) into eqs. (15)-(17), the equilibrium of moments gives

$$
\begin{aligned}
& \left(D_{11}+D_{\mathrm{f} 1}\right) \kappa_{x}+\left(D_{12}+v_{\mathrm{f}} D_{\mathrm{f} 1}\right) \kappa_{y}+2 D_{16} \kappa_{x y} \\
& \quad=R_{\mathrm{f} 1}\left[\varepsilon_{\mathrm{m} x}-\alpha_{\mathrm{f}} \Delta T+v_{\mathrm{f}}\left(\varepsilon_{\mathrm{m} y}-\alpha_{\mathrm{f}} \Delta T\right)\right], \\
& \left(D_{12}+v_{\mathrm{f}} D_{\mathrm{f} 1}\right) \kappa_{x}+\left(D_{22}+D_{\mathrm{f} 1}\right) \kappa_{y}+2 D_{26} \kappa_{x y} \\
& \quad=R_{\mathrm{f} 1}\left[\varepsilon_{\mathrm{m} y}-\alpha_{\mathrm{f}} \Delta T+v_{\mathrm{f}}\left(\varepsilon_{\mathrm{m} x}-\alpha_{\mathrm{f}} \Delta T\right)\right], \\
& D_{16} \kappa_{x}+D_{26} \kappa_{y}+2\left(D_{16}+D_{\mathrm{f} 2}\right) \kappa_{x y} \\
& \quad=R_{\mathrm{f} 2} \gamma_{\mathrm{m} x y},
\end{aligned}
$$

where

$$
\begin{aligned}
& D_{11}=\frac{B_{11} t_{\mathrm{s}}^{3}}{12}, \quad D_{12}=\frac{B_{12} t_{\mathrm{s}}^{3}}{12}, \quad D_{22}=\frac{B_{22} t_{\mathrm{s}}^{3}}{12}, \\
& D_{16}=\frac{B_{16} t_{\mathrm{s}}^{3}}{12}, \quad D_{26}=\frac{B_{26} t_{\mathrm{s}}^{3}}{12}, \quad D_{66}=\frac{B_{66} t_{\mathrm{s}}^{3}}{12}, \\
& D_{\mathrm{f} 1}=B_{\mathrm{f}}\left(\frac{t_{\mathrm{f}}^{3}}{3}+\frac{t_{\mathrm{f}}^{2} t_{\mathrm{s}}}{2}+\frac{t_{\mathrm{f}} t_{\mathrm{s}}^{2}}{4}\right), \quad D_{\mathrm{f} 2}=G_{\mathrm{f}}\left(\frac{t_{\mathrm{f}}^{3}}{3}+\frac{t_{\mathrm{f}}^{2} t_{\mathrm{s}}}{2}+\frac{t_{\mathrm{f}} t_{\mathrm{s}}^{2}}{4}\right), \\
& R_{\mathrm{f} 1}=\frac{B_{\mathrm{f}} t_{\mathrm{f}}\left(t_{\mathrm{f}}+t_{\mathrm{s}}\right)}{2}, \quad R_{\mathrm{f} 2}=\frac{G_{\mathrm{f}} t_{\mathrm{f}}\left(t_{\mathrm{f}}+t_{\mathrm{s}}\right)}{2} .
\end{aligned}
$$

When the coordinate axes coincide with the principal directions of elasticity, eqs. (21)-(26) become

$$
\begin{aligned}
& {\left[B_{11}\left(\varepsilon_{\mathrm{m} 1}-\alpha_{x}^{\mathrm{s}} \Delta T\right)+B_{12}\left(\varepsilon_{\mathrm{m} 2}-\alpha_{y}^{\mathrm{s}} \Delta T\right)\right] t_{\mathrm{s}}} \\
& \quad+B_{\mathrm{f}}\left[\left(\varepsilon_{\mathrm{m} 1}-\alpha_{\mathrm{f}} \Delta T\right)+v_{\mathrm{f}}\left(\varepsilon_{\mathrm{m} 2}-\alpha_{\mathrm{f}} \Delta T\right)\right] t_{\mathrm{f}}=0, \\
& \quad\left[B_{12}\left(\varepsilon_{\mathrm{m} 1}-\alpha_{x}^{\mathrm{s}} \Delta T\right)+B_{22}\left(\varepsilon_{\mathrm{m} 2}-\alpha_{y}^{\mathrm{s}} \Delta T\right)\right] t_{\mathrm{s}} \\
& \quad+B_{\mathrm{f}}\left[\left(\varepsilon_{\mathrm{m} 2}-\alpha_{\mathrm{f}} \Delta T\right)+v_{\mathrm{f}}\left(\varepsilon_{\mathrm{m} 1}-\alpha_{\mathrm{f}} \Delta T\right)\right] t_{\mathrm{f}}=0, \\
& \left(D_{11}+D_{\mathrm{f} 1}\right) \kappa_{1}+\left(D_{12}+v_{\mathrm{f}} D_{\mathrm{f} 1}\right) \kappa_{2} \\
& \quad=R_{\mathrm{f} 1}\left[\varepsilon_{\mathrm{m} 1}-\alpha_{\mathrm{f}} \Delta T+v_{\mathrm{f}}\left(\varepsilon_{\mathrm{m} 2}-\alpha_{\mathrm{f}} \Delta T\right)\right], \\
& \left(D_{12}+v_{\mathrm{f}} D_{\mathrm{f} 1}\right) \kappa_{1}+\left(D_{22}+D_{\mathrm{f} 1}\right) \kappa_{2} \\
& \quad=R_{\mathrm{f} 1}\left[\varepsilon_{\mathrm{m} 2}-\alpha_{\mathrm{f}} \Delta T+v_{\mathrm{f}}\left(\varepsilon_{\mathrm{m} 1}-\alpha_{\mathrm{f}} \Delta T\right)\right],
\end{aligned}
$$

where $\kappa_{1}$ and $\kappa_{2}$ are the principal curvatures, and $\varepsilon_{\mathrm{m} 1}$ and $\varepsilon_{\mathrm{m} 2}$ are the principal midplane strains. The principal midplane strains in eqs. (28) and (29) can be solved as

$$
\begin{aligned}
\varepsilon_{\mathrm{m} 1}= & r_{2}\left\{\left[r_{1} B_{\mathrm{f}}\left(B_{22}-B_{12} v_{\mathrm{f}}\right)+m_{1}\right] \kappa_{1}\right. \\
& \left.+\left[r_{1} B_{\mathrm{f}}\left(-B_{12}+B_{22} v_{\mathrm{f}}\right)+m_{2}\right] \kappa_{2}\right\}+\alpha_{x}^{\mathrm{s}} \Delta T, \\
\varepsilon_{\mathrm{m} 2}= & r_{2}\left\{\left[r_{1} B_{\mathrm{f}}\left(-B_{12}+B_{11} v_{\mathrm{f}}\right)+m_{3}\right] \kappa_{1}\right.
\end{aligned}
$$

$$
\left.+\left[r_{1} B_{\mathrm{f}}\left(B_{11}-B_{12} v_{\mathrm{f}}\right)+m_{4}\right] \kappa_{2}\right\}+\alpha_{y}^{\mathrm{s}} \Delta T,
$$

where

$m_{1}=D_{11} B_{22}-D_{12} B_{12}, \quad m_{2}=D_{12} B_{22}-D_{22} B_{12}$,

$m_{3}=D_{12} B_{11}-D_{11} B_{12}, \quad m_{4}=D_{22} B_{11}-D_{12} B_{12}$,

$$
r_{1}=\frac{t_{\mathrm{f}}^{3}}{3}+\frac{t_{\mathrm{f}}^{2} t_{\mathrm{s}}}{2}+\frac{t_{\mathrm{f}} t_{\mathrm{s}}^{2}}{4}, \quad r_{2}=\frac{-2}{t_{\mathrm{s}}\left(t_{\mathrm{f}}+t_{\mathrm{s}}\right)\left(B_{11} B_{22}-B_{12}^{2}\right)}
$$

Substituting eqs. (32) and (33) into eqs. (30) and (31), two relations containing the three film material constants $B_{\mathrm{f}}, v_{\mathrm{f}}$, and $\alpha_{\mathrm{f}}$ can be obtained:

$$
\begin{aligned}
\left(D_{11}\right. & \left.+r_{1} B_{\mathrm{f}}\right) \kappa_{1}+\left(D_{12}+r_{1} v_{\mathrm{f}} B_{\mathrm{f}}\right) \kappa_{2} \\
= & r_{1} r_{2} r_{3}\left(c_{1} \kappa_{1}+c_{2} \kappa_{2}\right) B_{\mathrm{f}}^{2} \\
& +r_{2} r_{3}\left[\left(m_{1}+v_{\mathrm{f}} m_{3}\right) \kappa_{1}+\left(m_{2}+v_{\mathrm{f}} m_{4}\right) \kappa_{2}\right] B_{\mathrm{f}} \\
& +r_{3}\left[\alpha_{x}^{\mathrm{s}}+v_{\mathrm{f}} \alpha_{y}^{\mathrm{s}}-\left(1+v_{\mathrm{f}}\right) \alpha_{\mathrm{f}}\right] B_{\mathrm{f}} \Delta T, \\
\left(D_{12}\right. & \left.+r_{1} v_{\mathrm{f}} B_{\mathrm{f}}\right) \kappa_{1}+\left(D_{22}+r_{1} B_{\mathrm{f}}\right) \kappa_{2} \\
= & r_{1} r_{2} r_{3}\left(c_{2} \kappa_{1}+c_{3} \kappa_{2}\right) B_{\mathrm{f}}^{2} \\
& +r_{2} r_{3}\left[\left(m_{3}+v_{\mathrm{f}} m_{1}\right) \kappa_{1}+\left(m_{4}+v_{\mathrm{f}} m_{2}\right) \kappa_{2}\right] B_{\mathrm{f}} \\
& +r_{3}\left[\alpha_{y}^{\mathrm{s}}+v_{\mathrm{f}} \alpha_{x}^{\mathrm{s}}-\left(1+v_{\mathrm{f}}\right) \alpha_{\mathrm{f}}\right] B_{\mathrm{f}} \Delta T,
\end{aligned}
$$

where

$$
\begin{aligned}
& r_{3}=\frac{t_{\mathrm{f}}\left(t_{\mathrm{f}}+t_{\mathrm{s}}\right)}{2}, \\
& c_{1}=B_{22}-2 B_{12} v_{\mathrm{f}}+B_{11} v_{\mathrm{f}}^{2}, \\
& c_{2}=-B_{12}+\left(B_{11}+B_{22}\right) v_{\mathrm{f}}-B_{12} v_{\mathrm{f}}^{2}, \\
& c_{3}=B_{11}-2 B_{12} v_{\mathrm{f}}+B_{22} v_{\mathrm{f}}^{2} .
\end{aligned}
$$

Equations (35) and (36) show that once the principal curvatures and one of the three film material constants are known, the determination of the other two film material constants is feasible. To facilitate the choice of constants to be fitted, a sensitivity analysis is carried out.

Since the given principal curvatures are preconditions for determining material constants, assigning a proper value to one of the three material constants whose variation negligibly affects the curvatures causes a small error in the determination of the other two material constants. Thus, carrying out a sensitivity analysis is helpful in forming a characterization strategy. The sensitivities of the principal curvatures $\kappa_{1}$ and $\kappa_{2}$ with respect to the film material constant $B_{\mathrm{f}}$ can be found from eqs. (35) and (36) as

$$
\frac{\partial \kappa_{1}}{\partial B_{\mathrm{f}}}=\frac{r_{1}\left(\kappa_{1}+v_{\mathrm{f}} \kappa_{2}\right)-r_{2} r_{3}\left[\left(m_{1}+m_{3} v_{\mathrm{f}}\right) \kappa_{1}+\left(m_{2}+m_{4} v_{\mathrm{f}}\right) \kappa_{2}\right]-2 r_{1} r_{2} r_{3}\left(c_{1} \kappa_{1}+c_{2} \kappa_{2}\right) B_{\mathrm{f}}-r_{3}\left[\alpha_{x}^{\mathrm{s}}+v_{\mathrm{f}} \alpha_{y}^{\mathrm{s}}-\left(1+v_{\mathrm{f}}\right) \alpha_{\mathrm{f}}\right] \Delta T}{-D_{11}-r_{1} B_{\mathrm{f}}+r_{2} r_{3}\left(m_{1}+m_{3} v_{\mathrm{f}}\right) B_{\mathrm{f}}+r_{1} r_{2} r_{3} c_{1} B_{\mathrm{f}}^{2}},
$$

and

$$
\frac{\partial \kappa_{2}}{\partial B_{\mathrm{f}}}=\frac{r_{1}\left(v_{\mathrm{f}} \kappa_{1}+\kappa_{2}\right)-r_{2} r_{3}\left[\left(m_{3}+m_{1} v_{\mathrm{f}}\right) \kappa_{1}+\left(m_{4}+m_{2} v_{\mathrm{f}}\right) \kappa_{2}\right]-2 r_{1} r_{2} r_{3}\left(c_{2} \kappa_{1}+c_{3} \kappa_{2}\right) B_{\mathrm{f}}-r_{3}\left[\alpha_{y}^{\mathrm{s}}+v_{\mathrm{f}} \alpha_{x}^{\mathrm{s}}-\left(1+v_{\mathrm{f}}\right) \alpha_{\mathrm{f}}\right] \Delta T}{-D_{22}-v_{\mathrm{f}} B_{\mathrm{f}}+r_{2} r_{3}\left(m_{4}+m_{2} v_{\mathrm{f}}\right) B_{\mathrm{f}}+r_{1} r_{2} r_{3} c_{3} B_{\mathrm{f}}^{2}}
$$

Similarly, the sensitivities of the curvatures with respect to $\nu_{\mathrm{f}}$ and $\alpha_{\mathrm{f}}$ can also be calculated as

$$
\begin{aligned}
\frac{\partial \kappa_{1}}{\partial \nu_{\mathrm{f}}} & =\frac{\left[r_{1} \kappa_{2}-r_{2} r_{3}\left(m_{3} \kappa_{1}+m_{4} \kappa_{2}\right)-r_{3} \alpha_{y}^{\mathrm{s}} \Delta T\right] B_{\mathrm{f}}-r_{1} r_{2} r_{3}\left(c_{4} \kappa_{1}+c_{5} \kappa_{2}\right) B_{\mathrm{f}}^{2}+r_{3} B_{\mathrm{f}} \alpha_{\mathrm{f}} \Delta T}{-D_{11}-r_{1} B_{\mathrm{f}}+r_{2} r_{3}\left(m_{1}+m_{3} \nu_{\mathrm{f}}\right) B_{\mathrm{f}}+r_{1} r_{2} r_{3} c_{1} B_{\mathrm{f}}^{2}}, \\
\frac{\partial \kappa_{2}}{\partial \nu_{\mathrm{f}}} & =\frac{\left[r_{1} \kappa_{1}-r_{2} r_{3}\left(m_{1} \kappa_{1}+m_{2} \kappa_{2}\right)-r_{3} \alpha_{x}^{\mathrm{s}} \Delta T\right] B_{\mathrm{f}}-r_{1} r_{2} r_{3}\left(c_{5} \kappa_{1}+c_{6} \kappa_{2}\right) B_{\mathrm{f}}^{2}+r_{3} B_{\mathrm{f}} \alpha_{\mathrm{f}} \Delta T}{-D_{22}-r_{1} B_{\mathrm{f}}+r_{2} r_{3}\left(m_{4}+m_{2} \nu_{\mathrm{f}}\right) B_{\mathrm{f}}+r_{1} r_{2} r_{3} c_{3} B_{\mathrm{f}}^{2}},
\end{aligned}
$$




$$
\frac{\partial \kappa_{1}}{\partial \alpha_{\mathrm{f}}}=\frac{\left(1+v_{\mathrm{f}}\right) r_{3} B_{\mathrm{f}} \Delta T}{-D_{11}-r_{1} B_{\mathrm{f}}+r_{2} r_{3}\left(m_{1}+m_{3} v_{\mathrm{f}}\right) B_{\mathrm{f}}+r_{1} r_{2} r_{3} c_{1} B_{\mathrm{f}}^{2}},
$$

and

$$
\frac{\partial \kappa_{2}}{\partial \alpha_{\mathrm{f}}}=\frac{\left(1+v_{\mathrm{f}}\right) r_{3} B_{\mathrm{f}} \Delta T}{-D_{22}-r_{1} B_{\mathrm{f}}+r_{2} r_{3}\left(m_{4}+m_{2} v_{\mathrm{f}}\right) B_{\mathrm{f}}+r_{1} r_{2} r_{3} c_{3} B_{\mathrm{f}}^{2}},
$$

where

$$
\begin{aligned}
& c_{4}=-2 B_{12}+2 B_{11} v_{\mathrm{f}}, \\
& c_{5}=B_{11}+B_{22}-2 B_{12} v_{\mathrm{f}}, \\
& c_{6}=-2 B_{12}+2 B_{22} v_{\mathrm{f}} .
\end{aligned}
$$

\section{Experimental Demonstration}

The developed technique is examined by depositing an aluminum film on an ST-cut quartz substrate. ST-cut quartz is a rotated $Y$-cut quartz with a $42.75^{\circ}$ cutting angle rotating about the crystalline $x$-axis. Its in-plane elastic constants can be calculated as $s_{11}^{\prime}=12.77 / \mathrm{TPa}\left(1 \mathrm{TPa}=10^{12} \mathrm{~N} \mathrm{~m}^{-2}\right)$, $s_{22}^{\prime}=9.792 / \mathrm{TPa}, \quad s_{12}^{\prime}=-2.693 / \mathrm{TPa}, \quad s_{16}^{\prime}=s_{26}^{\prime}=0$, and $s_{66}^{\prime}=15.40 / \mathrm{TPa}^{10)}$ The prime notation denotes the coordinate $x^{\prime}$ - and $y^{\prime}$-axes in the ST-cut quartz substrate that are transformed from the $x$ - and rotated $z$-axes of quartz crystal. The $x^{\prime}$-axis is also an axis of two-fold rotation symmetry.

The magnetron-sputtered aluminum film had a thickness of $0.22 \mu \mathrm{m}$ and the 4-in. ST-cut quartz substrate was $525 \mu \mathrm{m}$ thick. The deposition was carried out at $500{ }^{\circ} \mathrm{C}$ with an $\mathrm{Ar}$ pressure of $3 \mathrm{mTorr}$, a flow rate of $10 \mathrm{sccm}$, and a power of $50 \mathrm{~W}$. After deposition, the substrate was cooled to $27^{\circ} \mathrm{C}$. $\mathrm{X}$-ray diffraction (XRD) measurement shows that the aluminum film has a preferred (111) orientation, as shown in Fig. 2. The $2 \theta$ angles for the (111) and (200) orientations are 38.634 and $44.874^{\circ}$, which are larger than those of the aluminum film in zero stress state as 38.472 and $44.738^{\circ}$, respectively. ${ }^{11)}$ According to the preferred orientation and the shift in $2 \theta$ angle, the aluminum film is under tensile stress. ${ }^{12,13)}$ In addition to polycrystalline, this preferred orientation also has isotropic nature in the in-plane directions. $^{9)}$

The argon ion intrusion in the deposited film during sputtering may introduce intrinsic residual stress to the film. For low argon pressure and the target material whose atom mass is larger than that of argon, compressive film stress

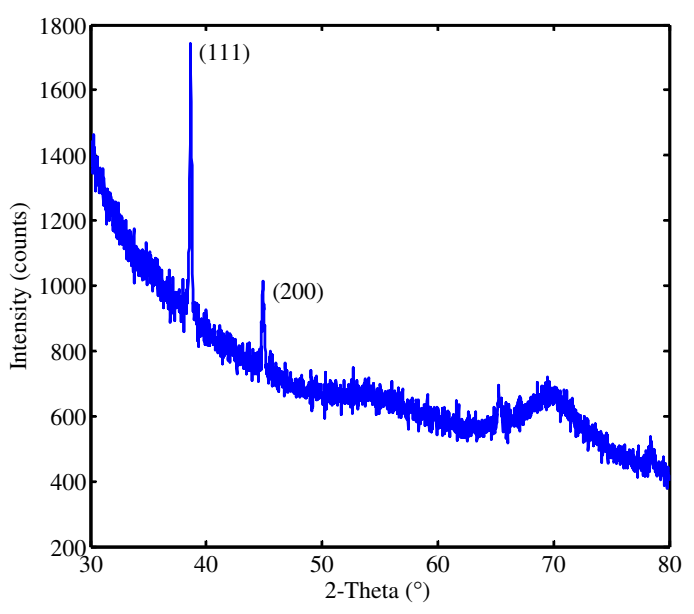

Fig. 2. (Color online) XRD pattern of sputtered aluminum film. develops owing to the bombardment of the reflected argon ions. However, for the aluminum film deposited below an argon pressure of $20 \mathrm{mTorr}$, the effect of ion bombardment on the deposited film is nearly negligible. ${ }^{12,14)}$ Energydispersive X-ray analysis (EDX) shows the bilayer-structure composition in weight percent (wt \%) as $49.5 \mathrm{wt} \%$ oxygen, $14.6 \mathrm{wt} \%$ aluminum, and $35.9 \mathrm{wt} \%$ silicon, which also confirms that argon is absent. Therefore, the deformation of the bilayer structure is mainly caused by thermal mismatch and the proposed characterization method can be adopted.

In reality, CTEs depend on the temperature $T$ and the deflection is due to the total thermal strain during deposition. The characterization method derived is based on this total thermal strain; therefore, it can only obtain the temperatureaveraged CTE $\bar{\alpha}$ defined as

$$
\bar{\alpha} \Delta T=\bar{\alpha}\left(T_{2}-T_{1}\right)=\int_{T_{1}}^{T_{2}} \alpha(T) d T,
$$

where $\alpha(T)$ is the instantaneous CTE, whereas $T_{1}$ to $T_{2}$ is the temperature history of the deposited film. The instantaneous CTEs of quartz from 0 to $500^{\circ} \mathrm{C}^{15)}$ can be fitted as

$$
\begin{aligned}
\alpha_{11 \text { (quartz) }}(T)= & 13.23+0.014 T-1.996 \times 10^{-5} T^{2} \\
& +4.519 \times 10^{-8} T^{3}, \\
\alpha_{33 \text { (quartz) }}(T)= & 7.009+0.0112 T-1.964 \times 10^{-5} T^{2} \\
+ & 3.602 \times 10^{-8} T^{3},
\end{aligned}
$$

where the units of $\alpha_{i j}$ and $T$ are $\mathrm{ppm} /{ }^{\circ} \mathrm{C}$ and ${ }^{\circ} \mathrm{C}$, respectively. Therefore, the averaged CTEs are $\bar{\alpha}_{11 \text { (quartz) }}=$ $16.6 \mathrm{ppm} /{ }^{\circ} \mathrm{C}$ and $\bar{\alpha}_{33 \text { (quartz) }}=9.43 \mathrm{ppm} /{ }^{\circ} \mathrm{C}$ accordingly. Consequently, the CTEs of the ST-cut quartz substrate can then be calculated as $\bar{\alpha}_{11 \text { (ST-cut) }}^{\prime}=16.6 \mathrm{ppm} /{ }^{\circ} \mathrm{C}$ and $\bar{\alpha}_{22 \text { (ST-cut) }}^{\prime}=12.76 \mathrm{ppm} /{ }^{\circ} \mathrm{C}$. The Young's modulus and Poisson's ratio of the bulk material for aluminum are $E_{\mathrm{Al}}=69 \mathrm{GPa}$ and $v_{\mathrm{Al}}=0.345$, respectively, ${ }^{16)}$ whereas its CTEs from 27 to $573^{\circ} \mathrm{C}^{17)}$ can be fitted as

$$
\begin{aligned}
\alpha_{\mathrm{Al}}(T)= & 15.54+0.0379 T-5.441 \times 10^{-5} T^{2} \\
& +4.444 \times 10^{-8} T^{3} .
\end{aligned}
$$

The averaged CTE of aluminum in this temperature range is therefore $\bar{\alpha}_{\mathrm{Al}}=27.4 \mathrm{ppm} /{ }^{\circ} \mathrm{C}$.

Because the characterization is based on the curvatures that cannot be measured directly, fitting the deflection data to find the curvatures is a priori. Therefore, finding a surface equation that can describe the deformation of the bilayer structure is necessary. Using the aforementioned material properties can obtain the midplane strains $\varepsilon_{\mathrm{m} x}, \varepsilon_{\mathrm{m} y}$, and $\varepsilon_{\mathrm{m} x y}$ from eqs. (21)-(23). Substituting these midplane strains into eqs. (24)-(26), the twist $\kappa_{x y}$ and curvatures $\kappa_{x}$ and $\kappa_{y}$ can be found. Having the thermomechanical properties in different in-plane directions been calculated for the anisotropic quartz substrate, the corresponding curvatures and twist are obtained and shown in Figs. 3(a) and 3(b), respectively. ${ }^{18)}$ 


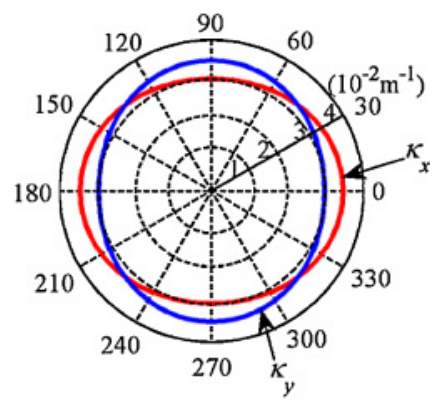

(a)

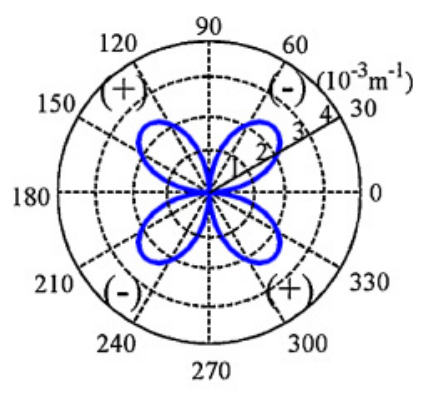

(b)
Fig. 3. (Color online) Calculated curvatures and twist for different in-plane crystalline orientations rotating about substrate normal: (a) curvatures $\kappa_{x}$ and $\kappa_{y}$, and (b) twist $\kappa_{x y}$. The directions of principal axes are 0 and $90^{\circ}$. The $0^{\circ}$ direction also corresponds to the axis of the two-fold rotation symmetry of the ST-cut quartz.

Figure 3 shows that there are two principal curvatures along the in-plane principal directions of substrate elasticity. This suggests a surface equation in the polar coordinate as

$$
\eta=a_{0}+a_{1} r+a_{2} r^{2}+a_{4} r^{4}
$$

with

$$
a_{n}=a_{n 0}+a_{n 2} \cos 2 \theta+b_{n 2} \sin 2 \theta, \quad n=1,2,4,
$$

where $\eta$ is the deflection, $r$ is the radial coordinate, $\theta$ is the azimuthal coordinate measured counterclockwise from the $x^{\prime}$-axis. Constant terms and the term of the first degree of $r$ are responsible for the rigid body motion, whereas terms of the second and fourth degrees of $r$ are employed, considering the linear symmetry of elastic deformation. Terms of $\cos 2 \theta$ and $\sin 2 \theta$ correspond to the two-fold rotation symmetry and the angular misalignment during measurement.

Contours were measured with a KLA-Tencor FLX-2320 system before and after the film deposition. The measurements were obtained at an angular increment of $5^{\circ}$ rotating about the substrate normal and with a range of $180^{\circ}$ to scan the entire substrate. The contour difference before and after the film deposition is the deflection due to thermal mismatch. Combining the measured data, contours for different stages and the substrate deflection are shown in Fig. 4. Using linear regression to fit the substrate deflection, the coefficients in eqs. (49) and (50) are determined as

$$
\begin{array}{ll}
a_{00}=-2.361 \times 10^{-5}, & a_{02}=4.144 \times 10^{-7}, \\
b_{02}=-2.948 \times 10^{-7}, & a_{10}=-4.166 \times 10^{-6}, \\
a_{12}=9.482 \times 10^{-6}, & b_{12}=-5.518 \times 10^{-6}, \\
a_{20}=1.610 \times 10^{-2}, & a_{22}=-9.546 \times 10^{-4}, \\
b_{22}=2.665 \times 10^{-4}, & a_{40}=-7.712 \times 10^{-1}, \\
a_{42}=2.760 \times 10^{-1}, & b_{42}=3.961 \times 10^{-2} .
\end{array}
$$

Theoretically, every location should have the same curvatures if the diameter of the bilayer structure is infinity. For a finite size substrate, linear distributions of strains along the $z$-axis as given in eq. (1) are not valid anywhere owing to boundary effects, with the consequence that the curvatures are varying with location. However, Saint-Vernant's principle ensures that the curvatures are almost constants and eq. (1) is a good approximation in the region far from the edge. Because of symmetry, the curvatures have their extrema at the center of the substrate and can be measured

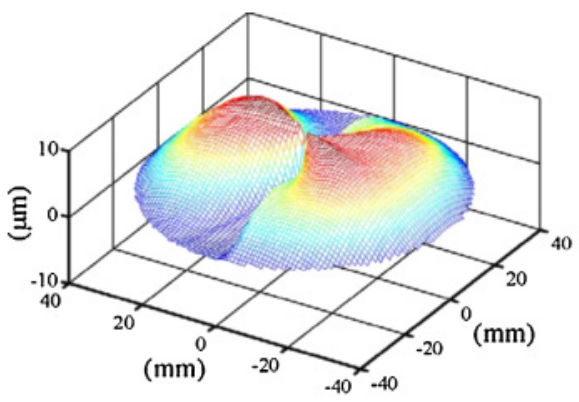

Before film deposition

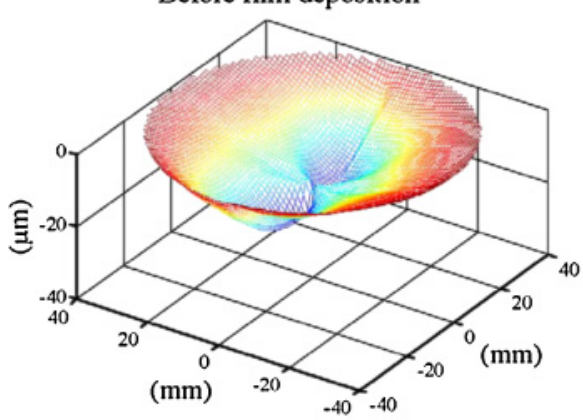

After film deposition

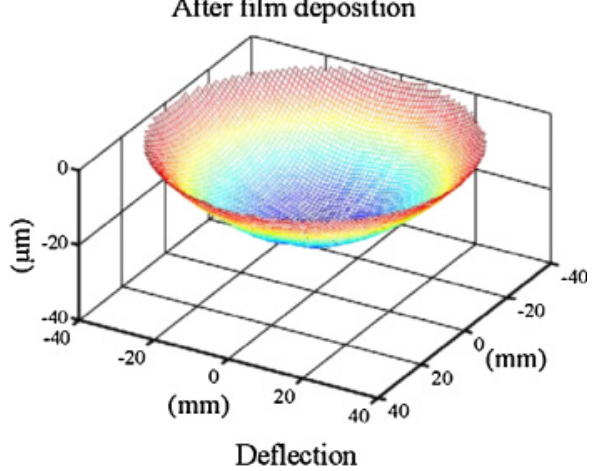

Fig. 4. (Color online) Three-dimensional contours for substrate before and after film deposition and its deflection.

more accurately than any location else. Therefore, the curvatures are calculated at $r=0$ with eq. (49) in different in-plane crystalline directions. These results are compared with those obtained from the theoretical model with thermomechanical properties of the bulk material, as shown in Fig. 5. The angle between the $x^{\prime}$-axis of ST-cut quartz and the direction for curvature measurement is the abscissa. The comparison range is chosen from 0 to $\pm 90^{\circ}$ with respect to the principal direction, as shown in Fig. 3. It is obvious that two curves have the same trend and the discrepancies are mainly due to the thermomechanical properties of the aluminum film being different from those of the bulk material.

The principal curvatures fitted are $\kappa_{1}=0.0342 \mathrm{~m}^{-1}$ and $\kappa_{2}=0.0302 \mathrm{~m}^{-1}$. Substituting these two principal curvatures and thermomechanical properties of bulk aluminum into eqs. (38)-(43), the corresponding sensitivities are

$$
\begin{aligned}
\partial \kappa_{1} / \partial B_{\mathrm{f}} & =4.54 \times 10^{-4} \mathrm{~m}^{-1} / \mathrm{GPa}, \\
\partial \kappa_{2} / \partial B_{\mathrm{f}} & =3.90 \times 10^{-4} \mathrm{~m}^{-1} / \mathrm{GPa}, \\
\partial \kappa_{1} / \partial v_{\mathrm{f}} & =3.30 \times 10^{-2} \mathrm{~m}^{-1}, \\
\partial \kappa_{2} / \partial v_{\mathrm{f}} & =1.79 \times 10^{-2} \mathrm{~m}^{-1}, \\
\partial \kappa_{1} / \partial \alpha_{\mathrm{f}} & =3.03 \times 10^{-3} \mathrm{~m}^{-1}{ }^{\circ} \mathrm{C} / \mathrm{ppm}, \\
\partial \kappa_{2} / \partial \alpha_{\mathrm{f}} & =2.24 \times 10^{-3} \mathrm{~m}^{-1}{ }^{\circ} \mathrm{C} / \mathrm{ppm},
\end{aligned}
$$




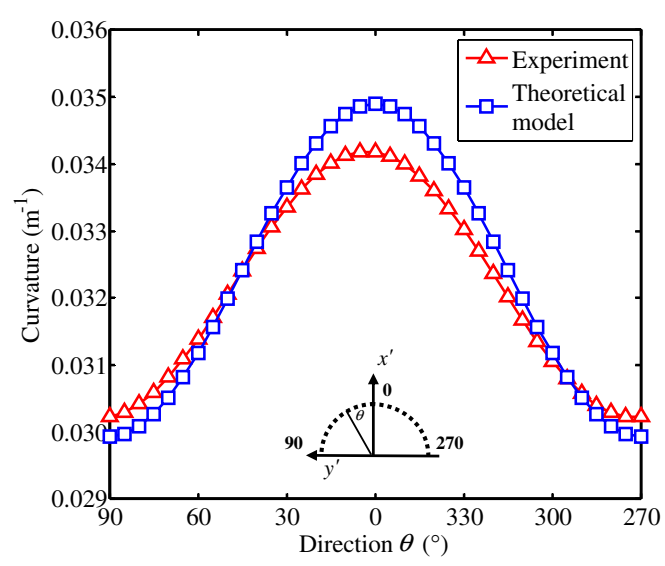

Fig. 5. (Color online) Curvatures obtained from theoretical model and experiment. The comparison range is chosen to be from 0 to $\pm 90^{\circ}$ with respect to the principal direction.

respectively. To determine the normalized sensitivities, a $1 \%$ variation is assigned to the bulk material constants, the corresponding changes in curvatures are approximately

$$
\begin{aligned}
& \Delta \kappa_{1}=\left(\partial k_{1} / \partial B_{\mathrm{f}}\right) \Delta B_{\mathrm{f}}=3.56 \times 10^{-4} \mathrm{~m}^{-1}, \\
& \Delta \kappa_{2}=\left(\partial k_{2} / \partial B_{\mathrm{f}}\right) \Delta B_{\mathrm{f}}=3.06 \times 10^{-4} \mathrm{~m}^{-1}, \\
& \Delta \kappa_{1}=\left(\partial k_{1} / \partial \nu_{\mathrm{f}}\right) \Delta \nu_{\mathrm{f}}=1.14 \times 10^{-4} \mathrm{~m}^{-1}, \\
& \Delta \kappa_{2}=\left(\partial k_{2} / \partial \nu_{\mathrm{f}}\right) \Delta \nu_{\mathrm{f}}=6.17 \times 10^{-5} \mathrm{~m}^{-1}, \\
& \Delta \kappa_{1}=\left(\partial k_{1} / \partial \alpha_{\mathrm{f}}\right) \Delta \alpha_{\mathrm{f}}=8.31 \times 10^{-4} \mathrm{~m}^{-1} \\
& \Delta \kappa_{2}=\left(\partial k_{2} / \partial \alpha_{\mathrm{f}}\right) \Delta \alpha_{\mathrm{f}}=6.15 \times 10^{-4} \mathrm{~m}^{-1}
\end{aligned}
$$

This indicates that the curvatures are relatively insensitive to the variation in Poisson's ratio. For the estimation of thin film material constants of aluminum, the Poisson's ratio is therefore assigned a value of 0.345 , the same as that of the bulk material. Subtracting eq. (35) from eq. (36) and plugging in the fitted principal curvatures, it results in a second-degree polynomial of $B_{\mathrm{f}}$

$$
B_{\mathrm{f}}^{2}+9.6362 \times 10^{16} B_{\mathrm{f}}-8.8875 \times 10^{27}=0 .
$$

This gives $B_{\mathrm{f}}$ a value of $92.23 \mathrm{GPa}$ whereas the second root is beyond the reasonable range. As a consequence, the evaluated Young's modulus and CTE of the aluminum film are $81.25 \mathrm{GPa}$ and $25.52 \mathrm{ppm} /{ }^{\circ} \mathrm{C}$, respectively.

Since the evaluated results depend on the preassigned Poisson's ratio, it is desired to investigate the effect of the variation in assigned value. Table I shows the evaluation results with respect to different Poisson's ratios. Differences of 2.57 and $3.40 \%$ for the evaluated Young's modulus and CTE are observed to correspond to a $10 \%$ variation in Poisson's ratio. This is consistent with the previous deduction.

Evaluation differences with respect to the variations in principal curvatures are also examined. The principal curvatures are altered by $\pm 3 \%$ to the previous fitted value. The maximum differences of 17.58 and $8.19 \%$ for the evaluated Young's modulus and CTE corresponding to $3 \%$ variations in principal curvatures are observed, as given in Table II. This shows that the evaluation of film material properties is very sensitive to the fitted curvatures. Therefore, it is very important to use a reasonable surface model to fit the deformation of the bilayer structure.
Table I. Evaluation of Young's modulus and CTE with respect to variations in Poisson's ratio.

\begin{tabular}{lccccc}
\hline \multirow{2}{*}{$\begin{array}{c}\text { Preassigned } \\
\text { Poisson's ratio }\end{array}$} & \multicolumn{2}{c}{ Young's modulus } & & \multicolumn{2}{c}{ CTE } \\
\cline { 2 - 3 } \cline { 5 - 6 } & $\begin{array}{c}\text { Evaluated } \\
(\mathrm{GPa})\end{array}$ & $\begin{array}{c}\text { Difference } \\
(\%)\end{array}$ & & $\begin{array}{c}\text { Evaluated } \\
\left(\mathrm{ppm} /{ }^{\circ} \mathrm{C}\right)\end{array}$ & $\begin{array}{c}\text { Difference } \\
(\%)\end{array}$ \\
\hline $0.3795\left(1.10 \nu_{\mathrm{b}}\right)$ & 83.34 & 2.57 & & 24.70 & -3.21 \\
$0.3622\left(1.05 v_{\mathrm{b}}\right)$ & 82.30 & 1.29 & & 25.11 & -1.60 \\
$0.345\left(v_{\mathrm{b}}\right.$, bulk value $)$ & 81.25 & N.A. & & 25.52 & N.A. \\
$0.3277\left(0.95 \nu_{\mathrm{b}}\right)$ & 80.21 & -1.28 & & 25.95 & 1.68 \\
$0.3105\left(0.90 \nu_{\mathrm{b}}\right)$ & 79.17 & -2.56 & & 26.39 & 3.40 \\
\hline
\end{tabular}

Table II. Evaluation of Young's modulus and CTE with respect to variations in principal curvatures

\begin{tabular}{cccccccc}
\hline \multicolumn{2}{c}{ Principal curvature } & & \multicolumn{2}{c}{ Young's modulus } & & \multicolumn{2}{c}{ CTE } \\
\cline { 1 - 2 } $\begin{array}{c}\kappa_{1} \\
\left(\mathrm{~m}^{-1}\right)\end{array}$ & $\begin{array}{c}\kappa_{2} \\
\left(\mathrm{~m}^{-1}\right)\end{array}$ & & $\begin{array}{c}\text { Evaluated } \\
(\mathrm{GPa})\end{array}$ & $\begin{array}{c}\text { Difference } \\
(\%)\end{array}$ & & $\begin{array}{c}\text { Evaluated } \\
\left(\mathrm{ppm} /{ }^{\circ} \mathrm{C}\right)\end{array}$ & $\begin{array}{c}\text { Difference } \\
(\%)\end{array}$ \\
\hline $0.0352(+3 \%)$ & 0.0302 & & 69.40 & -14.58 & & 27.54 & 7.91 \\
$0.0332(-3 \%)$ & 0.0302 & & 93.10 & 14.58 & & 24.02 & -5.88 \\
0.0342 & 0.0302 & & 81.25 & N.A. & & 25.52 & N.A. \\
0.0342 & $0.0311(+3 \%)$ & 95.54 & 17.58 & & 24.06 & -5.72 \\
0.0342 & $0.0293(-3 \%)$ & 66.97 & -17.58 & 27.61 & 8.19 \\
\hline
\end{tabular}

In the study of the Young's modulus of the sputtered aluminum film,${ }^{5)}$ the values from 77.6 to $80.9 \mathrm{GPa}$ and from 74.3 to $79.3 \mathrm{GPa}$ for thicknesses from 0.51 to $1.5 \mu \mathrm{m}$ were obtained by bending test and nanoindentation, respectively. Obviously, the results obtained by the proposed evaluation method are consistent with those of other studies using different techniques.

\section{Conclusions}

An on-wafer characterization method for the thermomechanical properties of a thin film has been proposed. Being deposited on an anisotropic substrate, the thermomechnical properties of a thin film can be determined from the deformation status of the bilayer structure. The lower the substrate symmetry, the more information of the thin film one can obtain. By employing a general orthotropic substrate, evaluating the Young's modulus and CTE of the film is possible. This method can only obtain a temperatureaveraged value for the temperature-dependent CTE.

An aluminum film deposited on an ST-cut quartz substrate was used for demonstration. A sensitivity analysis showed that the principal curvatures are relatively insensitive to the Poisson's ratio. Employing the Poisson's ratio of the bulk material, the Young's modulus and CTE of the aluminum film were determined to be $81.25 \mathrm{GPa}$ and $25.52 \mathrm{ppm} /{ }^{\circ} \mathrm{C}$, respectively. Simulation results show that the evaluation of film material properties is very sensitive to the fitted curvatures; therefore, a reasonable surface model for deformation fitting is crucial.

Further determination of the Poisson's ratio of the aluminum film is possible if the same film is also deposited on a substrate of different material. In this case, more equilibrium relations are obtained and the Young's modulus, CTE, and Poisson's ratio can be determined by solving the simultaneous equations. 


\section{Acknowledgement}

The authors thank Mr. Wei-Hsiang Chao at the Institute of Physics, Academia Sinica, for his assistance in XRD and EDX analyses.

1) S. Johansson, J.- $\AA$. Schweitz, L. Tenerz, and J. Tiren: J. Appl. Phys. 63 (1988) 4799.

2) S. Sundararajan, B. Bhushan, T. Namazu, and Y. Isono: Ultramicroscopy 91 (2002) 111.

3) M. G. Allen, M. Mehregany, R. T. Howe, and S. D. Senturia: Appl. Phys. Lett. 51 (1987) 241.

4) W. N. Sharpe, Jr., B. Yuan, and R. L. Edwards: J. Microelectromech. Syst. 6 (1997) 193.

5) D. Son, J. Jeong, and D. Kwon: Thin Solid Films 437 (2003) 182.

6) K. E. Petersen and C. R. Guarnieri: J. Appl. Phys. 50 (1979) 6761

7) T. F. Retajczyk and A. K. Sinha: Appl. Phys. Lett. 36 (1980) 161.

8) J.-H. Zhao, T. Ryan, P. S. Ho, A. J. McKerrow, and W.-Y. Shih: J.
Appl. Phys. 85 (1999) 6421.

9) J.-H. Zhao, Y. Du, M. Morgen, and P. S. Ho: J. Appl. Phys. 87 (2000) 1575 .

10) R. Bechman: Phys. Rev. 110 (1958) 1060.

11) H. E. Swanson and E. Tatge: U.S. Natl. Bur. Stand. Circ. 539, I (1953) 11

12) S. P. Kim, H. M. Choi, and S. K. Choi: Thin Solid Films 322 (1998) 298.

13) T. Namazu, S. Inoue, D. Ano, and K. Koterazawa: Proc. 17th IEEE Int. Conf., MEMS '04, 2004, p. 157.

14) K. Kamoshida: Thin Solid Films 283 (1996) 57.

15) R. B. Sosman: The Properties of Silica (Chemical Catalog, New York, 1927) p. 370.

16) M. M. de Lima, Jr., R. G. Lacerda, J. Vilcarromero, and F. C. Marques: J. Appl. Phys. 86 (1999) 4936.

17) J. F. Shackelford and W. Alexander: CRC Materials Science and Engineering Handbook (CRC, Boca Raton, FL, 2001) 3rd ed., Chap. 12.

18) S. G. Lekhnitskii, S. W. Tsai, and T. Cheron: Anisotropic Plates (Gordon and Breach, New York, 1968) Chap. 2. 